\title{
ON NON-HOLOMORPHIC FUNCTIONAL CALCULUS FOR COMMUTING OPERATORS
}

\author{
SEBASTIAN SANDBERG
}

\begin{abstract}
We provide a general scheme to extend Taylor's holomorphic functional calculus for several commuting operators to classes of non-holomorphic functions. These classes of functions will depend on the growth of the operator valued forms that define the resolvent cohomology class. The proofs are based on a generalization of the so-called resolvent identity to several commuting operators.
\end{abstract}

\section{Introduction}

Let $X, Y$ be two Banach spaces. We denote by $L(X, Y)$ the Banach space of all continuous linear operators from $X$ to $Y$ and we let $L(X)=L(X, X)$. We denote by $e$ the identity operator of $L(X)$. For a subset $A \subset L(X)$ we let $A^{\prime \prime}$ denote the bicommutant, that is the Banach algebra of all operators in $L(X)$ which commute with every operator in $\{b \in L(X): a b=b a$ for all $a \in A\}$.

Suppose that $a \in L(X)$. The spectrum of $a$ is defined as

$$
\sigma(a)=\{z \in \mathrm{C}: z-a \text { is not invertible }\},
$$

where $z-a$ is the operator $z e-a$. If $f$ is a holomorphic function in a neighborhood of $\sigma(a)$ then one can define the operator $f(a)$ by the integral

$$
f(a)=\frac{1}{2 \pi i} \int_{\partial D} f(z)(z-a)^{-1} d z,
$$

where $D$ is an appropriate neighborhood of $\sigma(a)$. This expression defines a continuous algebra homomorphism

$$
f \mapsto f(a): \mathscr{O}(\sigma(a)) \rightarrow(a)^{\prime \prime},
$$

such that $1(a)=e$ and $z(a)=a$, called the Riesz functional calculus. We want to extend this algebra homomorphism to functions not necessarily holomorphic

Received August 24, 2001. 
in a neighborhood of the spectrum. Following Dynkin [6] we define $f(a)$ by

$$
f(a)=-\frac{1}{2 \pi i} \int \bar{\partial} f(z) \wedge(z-a)^{-1} d z
$$

for all $f \in S_{a}$, where

$$
\bar{\partial} f=\frac{\partial f}{\partial \bar{z}} d \bar{z}
$$

and $S_{a}$ is defined by

$$
S_{a}=\left\{f \in C_{c}^{1}(\mathrm{C}):\|f\|_{a}:=\left\|\bar{\partial} f(z) \wedge(z-a)^{-1} d z\right\|_{\infty}<\infty\right\} .
$$

It is evident that $f(a)$ is a bounded linear operator on $X$ which commutes with each operator that commutes with $a$, that is $f(a) \in(a)^{\prime \prime}$. By Stokes theorem the definition of $f(a)$ only depends on the behavior of $f$ near $\sigma(a)$. Suppose that $D$ is an open set such that $\sigma(a) \subset D$ and that $f \in \mathscr{O}(D)$. Then if $\phi \in C_{c}^{1}(D)$ is equal to 1 in a neighborhood of $\sigma(a)$, we have that $\phi f \in S_{a}$ and $\phi f(a)$ defined by (1.2) equals $f(a)$ defined by (1.1).

We now prove that $f \mapsto f(a)$ is an algebra homomorphism and that the spectral mapping theorem holds for functions in $S_{a}$. This is done in Dynkin [6], but we provide proof as we will generalize these theorems to the several operator case.

THEOREM 1.1. The mapping

$$
f \mapsto f(a): S_{a} \rightarrow(a)^{\prime \prime},
$$

where $a \in L(X)$, is a continuous algebra homomorphism that continuously extends the holomorphic functional calculus. Moreover, if $f \in S_{a}$ then $\sigma(f(a))$ $=f(\sigma(a))$.

Proof. The map $f \mapsto f(a)$ is obviously linear and continuous. We have the so-called resolvent identity,

$$
(w-z)(z-a)^{-1}(w-a)^{-1}=(z-a)^{-1}-(w-a)^{-1}
$$

where $z, w \in \mathrm{C}$. The multiplicative property then follows,

$$
\begin{aligned}
f(a) & g(a) \\
= & \frac{1}{(2 \pi i)^{2}} \int_{z} \int_{w} \bar{\partial} f(z) \wedge(z-a)^{-1} d z \wedge \bar{\partial} g(w) \wedge(w-a)^{-1} d w \\
= & \frac{1}{(2 \pi i)^{2}} \int_{z} \int_{w} \bar{\partial} f(z) \wedge(z-a)^{-1} d z \wedge \bar{\partial} g(w) \wedge(w-z)^{-1} d w
\end{aligned}
$$




$$
\begin{aligned}
& +\frac{1}{(2 \pi i)^{2}} \int_{z} \int_{w} \bar{\partial} f(z) \wedge(z-w)^{-1} d z \wedge \bar{\partial} g(w) \wedge(w-a)^{-1} d w \\
=- & \frac{1}{2 \pi i} \int_{z} g(z) \bar{\partial} f(z) \wedge(z-a)^{-1} d z \\
& -\frac{1}{2 \pi i} \int_{w} f(w) \bar{\partial} g(w) \wedge(w-a)^{-1} d w=f g(a),
\end{aligned}
$$

by Fubini-Tonelli's theorem.

Suppose that $D$ is an open neighborhood of $\sigma(a)$ and that $f_{n} \in \mathscr{O}(D)$ is a sequence such that $f_{n} \rightarrow 0$ uniformly on compacts. Then if $\phi \in C_{c}^{1}(D)$ is a function equal to 1 in a neighborhood of $\sigma(a)$ we have that $\left\|f_{n} \phi\right\|_{a} \rightarrow 0$. Thus the mapping $f \mapsto f(a)$ continuously extends the holomorphic functional calculus.

If $w \notin f(\sigma(a))$ and $\phi \in C_{c}^{1}(\mathbf{C})$ is equal to 1 in an appropriate neighborhood of $g(\sigma(a))$, then

$$
\frac{\phi}{w-f} \in S_{a}
$$

and hence $w-f(a)$ is invertible and thus $w \notin \sigma(f(a))$. Therefore we have the inclusion $\sigma(f(a)) \subset f(\sigma(a))$. Suppose that $w \in f(\sigma(a))$ and assume that $w=0$. Then $0=f(\zeta)$ for some $\zeta \in \sigma(a)$. Let

$$
g(z)=\frac{f(z)}{z-\zeta} .
$$

Then

$$
\begin{aligned}
& f(a)=- \frac{1}{2 \pi i} \int_{z}(z-\zeta) \bar{\partial} g(z) \wedge(z-a)^{-1} d z \\
&=(\zeta-a) \frac{1}{2 \pi i} \int_{z} \bar{\partial} g(z) \wedge(z-a)^{-1} d z \\
& \quad-\frac{1}{2 \pi i} \int_{z}(z-a) \bar{\partial} g(z) \wedge(z-a)^{-1} d z .
\end{aligned}
$$

The last integral equals $f(\zeta)$, which is 0 , and hence $0 \in \sigma(f(a))$ since otherwise $\zeta-a$ would be invertible. Therefore $f(\sigma(a)) \subset \sigma(f(a))$, and hence the theorem is proved.

Furthermore, we have a rule of composition for this functional calculus.

THEOREM 1.2 (Rule of composition). If $g \in S_{a}$ and $f$ is a holomorphic function in a neighborhood of $\sigma(a)$, then $\phi(f \circ g) \in S_{a}$ and $f(g(a))=$ $\phi(f \circ g)(a)$, if $\phi \in C_{c}^{1}(\mathrm{C})$ is equal to 1 in a neighborhood of $\sigma(a)$. 
Proof. Suppose that $\psi \in C_{c}^{1}(\mathrm{C})$ is equal to 1 in a neighborhood of $\sigma(g(a))$. There is a function $\phi \in C_{c}^{1}(\mathrm{C})$ such that $\phi$ is equal to 1 in a neighborhood of $\sigma(a)$ and

$$
h=\frac{\phi}{w-g} \in S_{a}
$$

for each fixed $w \in \operatorname{supp}|\bar{\partial} \psi|$. The function $\phi(f \circ g)$ is in $S_{a}$ since

$$
\frac{\partial(\phi(f \circ g))}{\partial \bar{z}}=f \circ g \frac{\partial \phi}{\partial \bar{z}}+\phi \frac{\partial f}{\partial w} \frac{\partial g}{\partial \bar{z}} .
$$

Since

$$
\begin{aligned}
f(g(a)) & =-\frac{1}{2 \pi i} \int_{w} f(w) \bar{\partial}_{w} \psi(w) \wedge(w-g(a))^{-1} d w \\
& =\frac{1}{(2 \pi i)^{2}} \int_{w} \int_{z} f(w) \bar{\partial}_{w} \psi(w) \wedge d w \wedge \bar{\partial}_{z} h(z) \wedge(z-a)^{-1} d z \\
& =\frac{1}{(2 \pi i)^{2}} \int_{z} \bar{\partial}_{z} \int_{w} f(w) \bar{\partial}_{w} \psi(w) \wedge \frac{\phi(z) d w}{w-g(z)} \wedge(z-a)^{-1} d z \\
& =-\frac{1}{2 \pi i} \int_{z} \bar{\partial}_{z}(\phi f \circ g) \wedge(z-a)^{-1} d z=\phi(f \circ g)(a)
\end{aligned}
$$

the theorem is proved.

For further results regarding this functional calculus, see Dynkin [6].

Now we turn to the notion of spectrum of a commuting tuple of operators. Suppose that $a=\left(a_{1}, \ldots, a_{n}\right) \in L(X)^{n}$ is a commuting tuple of operators, that is $a_{i} a_{j}=a_{j} a_{i}$ for all $i$ and $j$. Denote by

$$
\Lambda=\oplus_{p=0}^{n} \Lambda^{p}
$$

the exterior algebra of $\mathrm{C}^{n}$ over $\mathrm{C}$. If $s_{1}, \cdots, s_{n}$ is a basis of $\mathrm{C}^{n}$ then $\Lambda$ has the basis

$$
s_{\emptyset}=1, \quad s_{I}=s_{i_{1}} \wedge \cdots \wedge s_{i_{p}}, \quad I=\left\{i_{1}, \cdots, i_{p}\right\},
$$

where $i_{1}<\cdots<i_{p}$ and $1 \leq p \leq n$, and we denote $\Lambda=\Lambda(s)$ in this case. We let $K_{\bullet}(a, X)$ be the Koszul complex induced by $a$,

$$
\cdots \rightarrow K_{p+1}(a, X) \stackrel{\delta_{p+1}}{\longrightarrow} K_{p}(a, X) \stackrel{\delta_{p}}{\longrightarrow} K_{p-1}(a, X) \rightarrow \cdots,
$$

where

$$
K_{p}(a, X)=\Lambda^{p}(s, X)=X \otimes c \Lambda^{p}(s)
$$


and

$$
\delta_{p}\left(x s_{I}\right)=2 \pi i \sum_{k=1}^{p}(-1)^{k-1} a_{i_{k}} x s_{i_{1}} \wedge \cdots \wedge \widehat{s}_{i_{k}} \wedge \cdots \wedge s_{i_{p}} .
$$

If $K_{\bullet}(a, X)$ is exact then $a$ is called nonsingular, otherwise singular. The spectrum is defined as

$$
\sigma(a)=\left\{z \in \mathrm{C}^{n}: z-a \text { is singular }\right\} .
$$

One also defines the split spectrum as

$$
\operatorname{sp}(a)=\left\{z \in \mathrm{C}^{n}: K_{\bullet}(z-a, X) \text { is not split }\right\},
$$

where split means that for every integer $p$ there are operators $h$ and $k$ such that $e=\delta_{p+1} h+k \delta_{p}$. If $X$ is a Hilbert space or $n=1$ then $\operatorname{sp}(a)=\sigma(a)$. In general we have that $\sigma(a) \subset \operatorname{sp}(a)$, but not the reverse inclusion, see Müller [11].

We will consider operators parametrized by a variable $z$, such as $z \mapsto z-a$. In that case the boundary map $\delta_{p}$ depends on $z$ and we will henceforth suppress the index $p$ and write $\delta_{p}$ as $\delta_{z-a}$ for every $p$. We also let $s_{i}=d z_{i}$.

Now suppose that $T \in L(X, Y)$ has closed range and let $k(T)$ be the norm of the inverse of $T$ considered as a map from $X / \operatorname{Ker} T$ to $\mathfrak{\Im} T$. The next lemma is Lemma 2.1.3 of [7], and it implies that if $a_{0}$ is a nonsingular tuple then $a$ is nonsingular if $\left\|a_{0}-a\right\|$ is small enough.

Lemma 1.3. Suppose that $X, Y, Z$ are Banach spaces, $\alpha_{0} \in L(X, Y)$, $\beta_{0} \in L(Y, Z), \Im \beta_{0}$ closed and $\operatorname{Ker} \beta_{0}=\Im \alpha_{0}$, that is

$$
X \stackrel{\alpha_{0}}{\longrightarrow} Y \stackrel{\beta_{0}}{\longrightarrow} Z
$$

is exact. Let $r$ be a number such that $r>\max \left\{k\left(\alpha_{0}\right), k\left(\beta_{0}\right)\right\}$. If $\alpha \in L(X, Y)$, $\beta \in L(Y, Z)$, ` $\alpha \subset \operatorname{Ker} \beta$ and $\left\|\alpha-\alpha_{0}\right\|,\left\|\beta-\beta_{0}\right\|<1 / 6 r$ then $\Im \alpha=\operatorname{Ker} \beta$ and $k(\alpha) \leq 4 r$.

Hence $\sigma(a)$ is closed. Furthermore, the spectrum has the projection property, see Theorem 2.5.4 of [7].

THEOREM 1.4. If $a \in L(X)^{n}$ and $a^{\prime}=\left(a, a_{n+1}\right) \in L(X)^{n+1}$ are commuting and $\pi: \mathrm{C}^{n+1} \rightarrow \mathrm{C}^{n}$ is defined by $\pi\left(z, z_{n+1}\right)=z$ then $\pi\left(\sigma\left(a^{\prime}\right)\right)=\sigma(a)$.

It follows that

$$
\sigma(a) \subset \sigma\left(a_{1}\right) \times \cdots \times \sigma\left(a_{n}\right)
$$

and hence $\sigma(a)$ is bounded. Thus $\sigma(a)$ is a compact subset of $C^{n}$. Conversely, any compact set $K$ in $C^{n}$ can arise as the spectrum of a commuting tuple of 
operators. This one sees by letting the operators $a_{k}$ to be multiplication by $z_{k}$ on the Banach space $C(K)$ of continuous functions on $K \subset \mathrm{C}^{n}$.

The next theorem says that pointwise exactness is equivalent to continuous exactness, see Corollary 2.1.4 of [7].

Theorem 1.5. Suppose that $X, Y, Z$ are Banach spaces and that $\Omega$ is a paracompact topological space. Furthermore suppose that $\alpha \in C(\Omega, L(X, Y))$ and $\beta \in C(\Omega, L(Y, Z))$ such that $\Im \beta(\lambda)$ is closed and $\operatorname{Ker} \beta(\lambda)=\Im \alpha(\lambda)$ for all $\lambda \in \Omega$. Then

$$
\operatorname{Ker}(C(\Omega, Y) \stackrel{\beta}{\rightarrow} C(\Omega, Z))=\Im(C(\Omega, X) \stackrel{\alpha}{\longrightarrow} C(\Omega, Y)) .
$$

Moreover for each point $\lambda \in \Omega$ and vector $x \in \operatorname{Ker} \alpha(\lambda)$ there is a function $f \in C(\Omega, X)$ with $\alpha f=0$ and $f(\lambda)=x$.

Thus the complex

$$
K_{\bullet}\left(a, C\left(C^{n} \backslash \sigma(a), X\right)\right)
$$

is exact. The next theorem is more complicated to prove, see Taylor [16], Theorem 2.16 and Eschmeier and Putinar [7], Section 6.4.

Theorem 1.6. Suppose that $U$ is an open subset of $\mathrm{C}^{n}, Y_{p}$ are Banach spaces, $\alpha_{p} \in \mathscr{O}\left(U, L\left(Y_{p}, Y_{p-1}\right)\right)$ and that

$$
\cdots \rightarrow Y_{p+1} \stackrel{\alpha_{p+1}(z)}{\longrightarrow} Y_{p} \stackrel{\alpha_{p}(z)}{\longrightarrow} Y_{p-1} \rightarrow \cdots
$$

is exact for all $z \in U$. Then the complex

$$
\cdots \rightarrow C^{\infty}\left(U, Y_{p+1}\right) \stackrel{\alpha_{p+1}}{\longrightarrow} C^{\infty}\left(U, Y_{p}\right) \stackrel{\alpha_{p}}{\longrightarrow} C^{\infty}\left(U, Y_{p-1}\right) \rightarrow \cdots
$$

is exact.

Hence the complex

$$
K_{\bullet}\left(a, C^{\infty}\left(C^{n} \backslash \sigma(a), X\right)\right)
$$

is exact.

This notion of joint spectrum for a commuting tuple of operator was introduced by Taylor, [15], in 1970. Furthermore, he proved the holomorphic functional calculus and the spectral mapping theorem for this spectrum in [16]. His first proof of the holomorphic functional calculus was based on the CauchyWeil integral. Using homological algebra he generalized the construction to not necessarily commuting tuples of operators in [18]. See Kisil and Ramirez de Arellano [9] for more recent developments of non-commuting functional calculus. In [1], [2] Andersson proved the holomorphic functional calculus for commuting operators using Cauchy-Fantappié-Leray formulas. 
The purpose of this paper is to study generalizations of Theorem 1.1 to the case of several commuting operators. Suppose that $E$ is a set such there is a smooth function $s$ such that $\delta_{z-a} s=e$ outside $E$. In that case we can use the integral representation from [1] to extend the holomorphic functional calculus. The main difficulty is to show the multiplication property; for this we will generalize the resolvent identity (1.3) to several commuting operators. In case $E$ is a convex set we can use approximation by holomorphic functions to show that the map $f \mapsto f(a)$ extends. Similar results to the one in this paper have been proved in Nguyen [13]. In the setting where one has a tuple $a$ of elements in a commutative Banach algebra (or more general a b-algebra) he extends the holomorphic functional calculus. The method of the proof of the multiplication property in [13] is to show that $f(a) g(a)=f \otimes g(T a)=$ $f \otimes g \circ T(a)=f g(a)$, where $T(z)=(z, z)$. In Droste [5] the holomorphic fuctional calculus is extended to ultradifferentiable functions in the case when the spectrum is contained in a totally real submanifold in $\mathrm{C}^{n}$. His method of proof is to use the denseness of the holomorphic functions in the algebra ultradifferentiable functions.

I would like to thank my supervisor Mats Andersson for valuable discussions about the results of this paper.

\section{Holomorphic functional calculus}

Remember that $X$ is a Banach space, $a \in L(X)^{n}$ is a tuple of commuting operators on $X$, and $z \in \mathrm{C}^{n}$ is a variable. Remember also the fact that if the complex $K_{\bullet}(z-a, X)$ is exact for every $z$ in an open set $U$ then there is a smooth solution $u$ in $U$ to the equation $\delta_{z-a} u=f$ if $f$ is a closed and smooth $X$-valued form in $U$.

We now construct the resolvent on $C^{n} \backslash \sigma(a)$. We remark that

$$
\delta_{z-a} \bar{\partial} \sum_{k} f_{k} d z_{k}=-2 \pi i \sum_{k, l}\left(z_{k}-a_{k}\right) \frac{\partial f_{k}}{\partial \bar{z}_{l}} d \bar{z}_{l}=-\bar{\partial} \delta_{z-a} \sum_{k} f_{k} d z_{k},
$$

and therefore $\delta_{z-a} \bar{\partial}=-\bar{\partial} \delta_{z-a}$ for 1-forms and hence for all forms since $\delta_{z-a}$ and $\bar{\partial}$ are anti-derivations. Suppose that $K_{\bullet}(z-a, X)$ is exact and $x \in X$. Then we can define a sequence $u_{i}$ in $\mathrm{C}^{n} \backslash \sigma(a)$ by

$$
\delta_{z-a} u_{1}=x, \quad \delta_{z-a} u_{i+1}=\bar{\partial} u_{i},
$$

since $\bar{\partial}$ and $\delta_{z-a}$ anti-commute. If this sequence starts with $x=0$ then there is a form $w_{n}$ such that $u_{n}=\bar{\partial} w_{n}$, this follows from the fact that we successively can find $w_{i}$ such that

$$
w_{1}=0, \quad \delta_{z-a} w_{i+1}=\bar{\partial} w_{i}-u_{i} .
$$


Thus if one has two sequences $u_{i}$ and $u_{i}^{\prime}$ as in (2.1) then the difference $u_{n}-u_{n}^{\prime}$ is exact. Hence $u_{n}$ defines a Dolbeault cohomology class $\omega_{z-a} x$ of bidegree $(n, n-1)$, which is called the resolvent cohomology class.

Suppose we have two cohomology classes, $\omega_{z-a} x$ and $\omega_{w-b} x$, where $z, w \in$ $C^{n}, a, b \in L(X)^{n}$, corresponding to sequences $u_{i}$ and $v_{i}$, respectively. Then one defines the $X$-valued cohomology class $\omega_{z-a} \wedge \omega_{w-b} x$ as the class of $c_{2 n}$, where $c_{i}$ solve

$$
c_{1}=0, \quad \delta_{z-a, w-b} c_{i+1}=\bar{\partial} c_{i}+v_{i}-u_{i} .
$$

To see that this really is a well defined cohomology class, let $u_{i}^{\prime}, v_{i}^{\prime}$ and $c_{i}^{\prime}$ be other choices of sequences. Let $w_{i}^{u}$ and $w_{i}^{v}$ be the sequences given by (2.2) for the sequences $u_{i}-u_{i}^{\prime}$ and $v_{i}-v_{i}^{\prime}$ respectively. Then we obtain

$$
c_{1}-c_{1}^{\prime}+w_{1}^{v}-w_{1}^{u}=0
$$

and

$$
\delta_{z-a, w-b}\left(c_{i+1}-c_{i+1}^{\prime}+w_{i+1}^{v}-w_{i+1}^{u}\right)=\bar{\partial}\left(c_{i}-c_{i}^{\prime}+w_{i}^{v}-w_{i}^{u}\right) .
$$

Hence, by (2.2) again, there exists a sequence $w_{i}^{c}$ such that $c_{2 n}-c_{2 n}^{\prime}=\bar{\partial} w_{2 n}^{c}$.

Now suppose that we instead have operator valued forms, $u_{i}$, such that

$$
\delta_{z-a} u_{1}=e, \quad \delta_{z-a} u_{i+1}=\bar{\partial} u_{i},
$$

so that $u_{n}$ represents the operator valued cohomology class $\omega_{z-a}$. Then we have that $\omega_{z-a} \wedge \omega_{w-b} x$ is the class of $u_{n} \wedge v_{n}$, where $v_{i}$ is an $X$-valued sequence defining $\omega_{w-b} x$. This follows from the fact

$$
\delta_{z-a}\left(u_{1} \wedge v_{n}\right)=v_{n}, \quad \delta_{z-a}\left(u_{i+1} \wedge v_{n}\right)=\bar{\partial}\left(u_{i} \wedge v_{n}\right)
$$

and the following proposition.

Proposition 2.1. If $v_{i}$ is a sequence defining $\omega_{w-b} x$ and

$$
\delta_{z-a} f_{1}=v_{n}, \quad \delta_{z-a} f_{i+1}=\bar{\partial} f_{i},
$$

then $f_{n}$ represents $\omega_{z-a} \wedge \omega_{w-b} x$.

Proof. Let $c_{i}$ be any sequence that defines $\omega_{z-a} \wedge \omega_{w-b} x$, so that $c_{i}$ satisfies (2.3). Denote by $c_{i}^{k, l}$ the component of $c_{i}$ which is of degree $k$ in $d z$ and degree $l$ in $d w$. We have that $\delta_{z-a} c_{i}^{0, i}=0$, so there is a form $f$ such that $c_{i}^{0,1}=\delta_{z-a} f$. This gives

$$
\delta_{z-a, w-b} c_{i}=\delta_{z-a, w-b}\left(c_{i}-c_{i}^{0,1}-\delta_{w-b} f\right),
$$


and hence we can assume that the component $c_{i}^{0, i}$ vanishes. We have that

$$
\delta_{z-a} c_{n+1}^{1, n}=v_{n}, \quad \delta_{z-a} c_{n+i+1}^{i+1, n}=\bar{\partial} c_{n+i}^{i, n},
$$

and therefore there is a form $w_{n}$ such that

$$
f_{n}-c_{2 n}^{n, n}+\bar{\partial} w_{n}=0 .
$$

Since $c_{2 n}=c_{2 n}^{n, n}$ the proposition is proved.

In one variable there is only one possible representative for $\omega_{z-a} x, a \in$ $L(X)$,

$$
\omega_{z-a} x=\frac{1}{2 \pi i}(z-a)^{-1} d z x,
$$

and we have that $\omega_{z-a}$ is operator valued. The key part of the proof of the holomorphic functional calculus in one variable is the resolvent identity (1.3), which we can reformulate as

$$
\omega_{z-a} \wedge \omega_{w-a}+\omega_{w-a} \wedge \omega_{z-w}+\omega_{w-z} \wedge \omega_{z-a}=0 .
$$

We will now generalize this equality to several commuting operators. Let $\Delta=$ $\left\{(z, w) \in \mathrm{C}^{2 n}: z=w\right\}$ be the diagonal.

Lemma 2.2. For every $x \in X$, we have the equality

$$
\omega_{z-a} \wedge \omega_{w-a} x+\omega_{w-a} \wedge \omega_{z-w} x+\omega_{w-z} \wedge \omega_{z-a} x=0,
$$

on $\left(\left(\mathrm{C}^{n} \backslash \sigma(a)\right) \times \mathrm{C}^{n} \cap \mathrm{C}^{n} \times\left(\mathrm{C}^{n} \backslash \sigma(a)\right)\right) \backslash \Delta$.

Proof. Define the sequence $m_{k}$ by

$$
m_{k}=\frac{1}{(2 \pi i)^{k}} \frac{\partial|z-w|^{2}}{|z-w|^{2}} \wedge\left(\bar{\partial} \frac{\partial|z-w|^{2}}{|z-w|^{2}}\right)^{k-1} .
$$

The equalities,

$$
\begin{aligned}
\delta_{z-a, w-a} m_{1} & =\frac{1}{2 \pi i|z-w|^{2}} \delta_{z-a, w-a} \partial|z-w|^{2}=1, \\
\delta_{z-a, w-a} m_{k+1} & =\frac{1}{(2 \pi i)^{k}}\left(\bar{\partial} \frac{\partial|z-w|^{2}}{|z-w|^{2}}\right)^{k}=\bar{\partial} m_{k},
\end{aligned}
$$

for all $k \leq n$, and $m_{k}=0$ for all $k>n$, hold on $C^{2 n} \backslash \Delta$. Let $u_{i}$ be a sequence as in (2.1) that defines $\omega_{z-a} x$. Define $u_{i}^{1}$ and $u_{i}^{2}$ by $u_{i}^{1}=\pi_{1}^{*} u_{i}$ and $u_{i}^{2}=\pi_{2}^{*} u_{i}$, 
where $\pi_{1}(z, w)=z$ and $\pi_{2}(z, w)=w$ are the projections. Let $c_{i}$ be a sequence that satisfies the equalities

$$
c_{1}=0, \quad \delta_{z-a, w-a} c_{l+1}=\bar{\partial} c_{l}+u_{l}^{2}-u_{l}^{1} .
$$

Using the equalities (2.7), (2.8) and (2.9) (for $l \geq n$ ), we get

$$
\begin{aligned}
&-\bar{\partial} \sum_{k+l=2 n} m_{k} \wedge c_{l}= \delta_{z-a, w-a} \sum_{k+l=2 n+1} m_{k} \wedge c_{l}-\bar{\partial} \sum_{k+l=2 n} m_{k} \wedge c_{l} \\
&= \sum_{l=n+1}^{2 n} \delta_{z-a, w-a} m_{2 n+1-l} \wedge c_{l}-\sum_{l=n}^{2 n-1} \bar{\partial} m_{2 n-l} \wedge c_{l} \\
& \quad+\sum_{k=1}^{n} m_{k} \wedge\left(\bar{\partial} c_{2 n-k}-\delta_{z-a, w-a} c_{2 n+1-k}\right) \\
&=-\bar{\partial} m_{n} \wedge c_{n}+c_{2 n}+m_{n} \wedge\left(u_{n}^{1}-u_{n}^{2}\right) .
\end{aligned}
$$

Thus

$$
-\bar{\partial} \sum_{k+l=2 n} m_{k} \wedge c_{l}=c_{2 n}+u_{n}^{2} \wedge m_{n}+m_{n} \wedge u_{n}^{1}
$$

outside the diagonal. We have that the component of $m_{n}$ which does not contain $d w$ and $d \bar{w}$ represents $\omega_{z-w}$ and that the component of $m_{n}$ which does not contain $d z$ or $d \bar{z}$ represents $\omega_{w-z}$. Since $c_{2 n}$ represents $\omega_{z-a} \wedge \omega_{w-a} x$, the lemma follows from (2.10).

Choose representatives $\widetilde{\omega}_{z-a} x, \widetilde{\omega}_{w-a} x$ and $\widetilde{\omega}_{z-a} \wedge \widetilde{\omega}_{w-a} x$ for $\omega_{z-a} x, \omega_{w-a} x$ and $\omega_{z-a} \wedge \omega_{w-a} x$ respectively on $\left(\mathrm{C}^{n} \backslash \sigma(a)\right) \times \mathrm{C}^{n} \cap \mathrm{C}^{n} \times\left(\mathrm{C}^{n} \backslash \sigma(a)\right)$. Let $\widetilde{\omega}_{z-w}=m_{n}$. Then (2.5) says that the form

$$
\widetilde{\omega}_{z-a} \wedge \widetilde{\omega}_{w-a} x+\widetilde{\omega}_{w-a} \wedge \widetilde{\omega}_{z-w} x+\widetilde{\omega}_{z-w} \wedge \widetilde{\omega}_{z-a} x,
$$

defined on $\left(\left(\mathrm{C}^{n} \backslash \sigma(a)\right) \times \mathrm{C}^{n} \cap \mathrm{C}^{n} \times\left(\mathrm{C}^{n} \backslash \sigma(a)\right)\right) \backslash \Delta$, is exact. We want this expression to be an exact current over $\Delta$ as well. Suppose that (2.11) is exact on $\left(\mathrm{C}^{n} \backslash \sigma(a)\right) \times \mathrm{C}^{n} \cap \mathrm{C}^{n} \times\left(\mathrm{C}^{n} \backslash \sigma(a)\right)$. We have $[\Delta]=\bar{\partial} \widetilde{\omega}_{z-w}$, where $[\Delta]$ denotes the current of integration over $\Delta$. If we apply $\bar{\partial}$ to (2.11), interpreted as a current, we obtain

$$
0=-\widetilde{\omega}_{w-a} x \wedge[\Delta]+[\Delta] \wedge \widetilde{\omega}_{z-a} x=[\Delta] \wedge\left(\widetilde{\omega}_{z-a} x-\widetilde{\omega}_{w-a} x\right)
$$

since (2.11) is supposed to be exact and therefore is closed. Hence $i^{*}\left(\widetilde{\omega}_{z-a} x-\right.$ $\left.\widetilde{\omega}_{w-a} x\right)=0$, where $i$ is a function defined by $i(\tau)=(\tau, \tau)$. The next theorem gives the desired result in the case where we have $i^{*} \widetilde{\omega}_{z-a} x=i^{*} \widetilde{\omega}_{w-a} x$. 
TheOREM 2.3 (Resolvent identity). Suppose that $\widetilde{\omega}_{z-a} x, \widetilde{\omega}_{w-a} x$ and $\widetilde{\omega}_{z-a} \wedge$ $\widetilde{\omega}_{w-a} x$ are representatives for $\omega_{z-a} x, \omega_{w-a} x$ and $\omega_{z-a} \wedge \omega_{w-a} x$, respectively. Let $\widetilde{\omega}_{z-w}=m_{n}$, where $m_{n}$ is defined in (2.6). Then the current

$$
\widetilde{\omega}_{z-a} \wedge \widetilde{\omega}_{w-a} x+\widetilde{\omega}_{w-a} \wedge \widetilde{\omega}_{z-w} x+\widetilde{\omega}_{z-w} \wedge \widetilde{\omega}_{z-a} x
$$

defined on $\left(\mathrm{C}^{n} \backslash \sigma(a)\right) \times \mathrm{C}^{n} \cap \mathrm{C}^{n} \times\left(\mathrm{C}^{n} \backslash \sigma(a)\right)$ is exact if and only if $i^{*} \widetilde{\omega}_{z-a} x=$ $i^{*} \widetilde{\omega}_{w-a} x$, where $i: \mathrm{C}^{n} \rightarrow \mathrm{C}^{2 n}$ is defined by $i(\tau)=(\tau, \tau)$.

Proof. The necessity of having $i^{*}\left(\widetilde{\omega}_{z-a} x-\widetilde{\omega}_{w-a} x\right)=0$ has already been proved. Now suppose that $i^{*}\left(\widetilde{\omega}_{z-a} x-\widetilde{\omega}_{w-a} x\right)=0$. Let $u_{i}^{1}, u_{i}^{2}, m_{i}$ and $c_{i}$ be the sequences in the proof of Lemma 2.2. Let $\delta=\delta_{z-a, w-a}$. Then we have $i^{*} \delta=\delta_{\tau-a} i^{*}$ by induction, since

$$
\begin{aligned}
i^{*} \delta\left(f d z_{k}+g d w_{l}\right) & =\left(\tau_{k}-a_{k}\right) f(\tau, \tau)+\left(\tau_{l}-a_{l}\right) g(\tau, \tau) \\
& =\delta_{\tau-a} i^{*}\left(f d z_{k}+g d w_{l}\right)
\end{aligned}
$$

and

$$
i^{*} \delta(u \wedge v)=i^{*} \delta u \wedge i^{*} v-i^{*} u \wedge i^{*} \delta v=\delta_{\tau-a} i^{*}(u \wedge v),
$$

if $u$ is a 1 -form. Thus

$$
i^{*} c_{1}=0, \quad \delta_{\tau-a} i^{*} c_{i+1}=\bar{\partial} i^{*} c_{i}
$$

and hence, by (2.2), there is a form $w_{n}$ of $\tau$ such that $i^{*} c_{n}=\bar{\partial} w_{n}$. For all test forms $f$ we have the identity

$$
\bar{\partial} m_{n} \wedge c_{n} . f=\int_{\Delta} i^{*}\left(c_{n} \wedge f\right)=\int_{\Delta} \bar{\partial} w_{n} \wedge i^{*} f=\int_{\Delta} w_{n} \wedge i^{*} \bar{\partial} f .
$$

Therefore the calculation in the proof of Lemma 2.2 gives the equality

$$
-\bar{\partial}\left([\Delta] \wedge w_{n}+\sum_{k=1}^{n} m_{k} \wedge c_{2 n-k}\right)=c_{2 n}+u_{n}^{2} \wedge m_{n}+m_{n} \wedge u_{n}^{1} .
$$

Since $\widetilde{\omega}_{\tau-a} x$ and $u_{n}$ represent the same cohomology class, there is a form $q$ such that $\widetilde{\omega}_{\tau-a} x-u_{n}=\bar{\partial} q$. Let $q^{1}=\pi_{1}^{*} q$ and $q^{2}=\pi_{2}^{*} q$. Then

$$
\begin{aligned}
& \widetilde{\omega}_{z-w} \wedge\left(\widetilde{\omega}_{z-a} x-\widetilde{\omega}_{w-a} x-\left(u_{n}^{1}-u_{n}^{2}\right)\right)=\widetilde{\omega}_{z-w} \wedge\left(\bar{\partial} q^{1}-\bar{\partial} q^{2}\right) \\
&=[\Delta] \wedge\left(q^{1}-q^{2}\right)-\bar{\partial}\left(\widetilde{\omega}_{z-w} \wedge\left(q^{1}-q^{2}\right)\right)=-\bar{\partial}\left(\widetilde{\omega}_{z-w} \wedge\left(q^{1}-q^{2}\right)\right) .
\end{aligned}
$$

Thus, since $\widetilde{\omega}_{z-a} \wedge \widetilde{\omega}_{w-a} x-c_{2 n}$ is an exact current, the theorem is proved. 
Now we give the definition of $f(a)$. If $f$ is a holomorphic function in a neighborhood of $\sigma(a)$ then we define $f(a)$ by the formula

$$
f(a) x=-\int f \bar{\partial} \phi \wedge \omega_{z-a} x \quad \text { for all } \quad x \in X,
$$

where $\phi \in C_{c}^{\infty}$ is equal to 1 in a neighborhood of $\sigma(a)$. This definition is independent of the choice of $\phi$. To see this, suppose that $\varphi \in C_{c}^{\infty}$ is equal to 0 in a neighborhood of the spectrum. Then we obtain

$$
\int \bar{\partial} \varphi \wedge \omega_{z-a} x=\int \bar{\partial} \varphi \wedge u_{n}=\int \bar{\partial}\left(\varphi \wedge u_{n}\right)=0,
$$

if $u_{n}$ is a smooth form in $C^{n} \backslash \sigma(a)$ representing $\omega_{z-a} x$. Note also that, by Stokes theorem, we have the equality

$$
-\int f \bar{\partial} \phi \wedge \omega_{z-a} x=\int_{\partial D} f \omega_{z-a} x
$$

where $D$ is a small enough neighborhood of $\sigma(a)$. We now prove that $f(a) \in$ $(a)^{\prime \prime}$.

Lemma 2.4. If $f(a)$ is defined by the formula (2.13), then $f(a) \in(a)^{\prime \prime}$.

Proof. Suppose that $x, y \in X$ and $c, d \in \mathrm{C}$. Denote by $u_{i}^{x}$ the sequence (2.1). Then

$$
\delta_{z-a}\left(u_{1}^{c x+d y}-c u_{1}^{x}-d u_{1}^{y}\right)=0
$$

and

$$
\delta_{z-a}\left(u_{i+1}^{c x+d y}-c u_{i+1}^{x}-d u_{i+1}^{y}\right)=\bar{\partial}\left(u_{i}^{c x+d y}-c u_{i}^{x}-d u_{i}^{y}\right),
$$

so $u_{n}^{c x+d y}$ and $c u_{n}^{x}+d u_{n}^{y}$ define the same cohomology class. Therefore the resolvent is linear, i.e.,

$$
\omega_{z-a}(c x+d y)=c \omega_{z-a} x+d \omega_{z-a} y,
$$

and hence $f(a)$ is a linear operator.

The map $\delta_{z-a}$ is linear, continuous and surjective between the Frechet space of all $C_{p+1, q}^{\infty}(U, X)$ forms to the Frechet space of all $\delta_{z-a}$-closed $C_{p, q}^{\infty}(U, X)$ forms, where $U=\mathrm{C}^{n} \backslash \sigma(a)$. Let $K_{1} \subset \mathrm{C}^{n} \backslash \sigma(a)$ be a given compact set and let $t_{1}=0$. The open mapping theorem gives the existence of a sequence of compact sets $K_{i} \subset \mathrm{C}^{n} \backslash \sigma(a)$ and natural numbers $t_{i}$ such that the equation $\delta_{z-a} u=v$ has a solution $u$, which satisfies

$$
\|u\|_{K_{i}, t_{i}+1} \leq C\|v\|_{K_{i+1}, t_{i+1}}
$$


for all closed $v$. Thus we can choose the sequence (2.1) so that

$$
\left\|u_{1}\right\|_{K_{n}, t_{n}+1} \leq C\|x\|_{K_{n+1}, t_{n+1}}=C\|x\|
$$

and

$$
\left\|u_{i+1}\right\|_{K_{n-i}, t_{n-i}+1} \leq C\left\|\bar{\partial} u_{i}\right\|_{K_{n-i+1}, t_{n-i+1}} \leq C\left\|u_{i}\right\|_{K_{n-i+1}, t_{n-i+1}+1} .
$$

Hence

$$
\|f(a) x\| \leq \int\left\|f \bar{\partial} \phi \wedge u_{n}\right\| \leq C|f|_{\text {supp } \phi}\|x\|
$$

and thus the operator $f(a)$ is bounded.

Suppose that $b \in L(X)$ is an operator which commutes with the tuple $a$. Then

$$
\delta_{z-a} b u_{1}^{x}=b x, \quad \delta_{z-a} b u_{i+1}^{x}=\bar{\partial} b u_{i}^{x},
$$

so $b u_{n}^{x}$ and $u_{n}^{b x}$ defines the same cohomology class. Therefore

$$
b \omega_{z-a} x=\omega_{z-a} b x
$$

and thus $f(a) \in(a)^{\prime \prime}$.

We can now prove Taylor's theorem.

Theorem 2.5 (Taylor). The mapping

$$
f \mapsto f(a): \mathscr{O}(\sigma(a)) \rightarrow(a)^{\prime \prime}
$$

is a continuous algebra homomorphism such that $1(a)=e$ and $z_{k}(a)=a_{k}$.

Proof. The map $f \mapsto f(a)$ is continuous by (2.14). We now prove that $f(a) g(a)=f g(a)$. Let $u_{i}, u_{i}^{1}, u_{i}^{2}$ and $c_{i}$ be as in Lemma 2.2. By the proof of Proposition 2.1 we can assume that the component $c_{i}^{0, i}$ vanishes. Since

$$
\delta_{z-a} c_{n+1}^{1, n}=u_{n}(w), \quad \delta_{z-a} c_{n+i+1}^{i+1, n}=\bar{\partial} c_{n+i}^{i, n},
$$

we have that $c_{2 n}$ represents $\omega_{z-a} u_{n}(w)$ and thus we have that

$$
f(a) u_{n}(w)=-\int_{z} f(z) \bar{\partial} \phi_{1}(z) \wedge \omega_{z-a} u_{n}(w)=-\int_{z} f(z) \bar{\partial} \phi_{1}(z) \wedge c_{2 n} .
$$

Multiplying this equality by $g(w) \bar{\partial} \phi_{2}(w)$ and integrating with respect to $w$ we get

$$
f(a) g(a) x=\int_{w} \int_{z} f(z) g(w) \bar{\partial} \phi_{2}(w) \wedge \bar{\partial} \phi_{1}(z) \wedge c_{2 n} .
$$


The resolvent identity (2.12) then gives that the right hand side is equal to

$$
\iint f g \bar{\partial} \phi_{1} \wedge \bar{\partial} \phi_{2} \wedge m_{n} \wedge u_{n}^{1}+\iint f g \bar{\partial} \phi_{1} \wedge \bar{\partial} \phi_{2} \wedge u_{n}^{2} \wedge m_{n},
$$

and hence we get, by the Bochner-Martinelli integral formula,

$$
-\int\left(f g \phi_{2} \bar{\partial} \phi_{1}+f \phi_{1} g \bar{\partial} \phi_{2}\right) \wedge u_{n}=-\int f g \bar{\partial}\left(\phi_{1} \phi_{2}\right) \wedge u_{n}=f g(a) x,
$$

since $u_{n}^{1}=\pi_{1}^{*} u_{n}$ and $u_{n}^{2}=\pi_{2}^{*} u_{n}$. Since the map (2.15) obviously is linear, it is an algebra homomorphism.

It remains to prove that $1(a)=e$ and $z_{k}(a)=a_{k}$. The first equality follows by representing $\omega_{z-a}$ by

$$
\frac{1}{(2 \pi i)^{n}}\left(|z|^{2} e-\bar{z} a\right)^{-n} \partial|z|^{2} \wedge\left(\bar{\partial} \partial|z|^{2}\right)^{n-1},
$$

cf. [1], and integrating against $\bar{\partial} \phi$, where $\phi$ is a radial cutoff function which is equal to 1 in a neighborhood of $\sigma(a)$. The second equality follows from the first equality and the equalities

$$
\left(z_{k}-a_{k}\right) u_{n}=\frac{1}{2 \pi i}\left(\delta_{z-a} u_{n}\right) \wedge d z_{k}=\frac{1}{2 \pi i} \bar{\partial}\left(u_{n-1} \wedge d z_{k}\right),
$$

where $u_{i}$ is a sequence that satisfies (2.1).

Taylor also proved the spectral mapping theorem; if $f \in \mathscr{O}(\sigma(a))$ then $f(\sigma(a))=\sigma(f(a))$. Suppose that $a$ is a commuting tuple and that $D$ is an open set such that $\sigma(a) \subset D$. Then there exists a $\delta>0$ such that $\sigma(b) \subset D$ if $\|a-b\|<\delta$. This follows from Lemma 1.3. In Newburgh [12] it is proved that the spectrum of one operator is continuous under commutative perturbations; the next proposition says that the same is true for the Taylor spectrum.

Proposition 2.6. If $a$ and $b$ are tuples of operators such that $a, b$ is commuting then

$$
\sup _{z \in \sigma(a)} \inf _{w \in \sigma(b)}|z-w|+\sup _{w \in \sigma(b)} \inf _{z \in \sigma(a)}|z-w| \leq 2 \sup _{z \in \sigma(a-b)}|z| \leq 2\|a-b\| .
$$

Proof. Suppose that $u \in \sigma(a)$. Since $P \sigma(a, b)=\sigma(a)$, where $P(z, w)=$ $z$, there is a $v$ in $\sigma(b)$ such that $(u, v) \in \sigma(a, b)$. Since $T \sigma(a, b)=\sigma(a-b)$, where $T(z, w)=z-w$, we have that $u-v \in \sigma(a-b)$. Thus

$$
\sup _{z \in \sigma(a)} \inf _{w \in \sigma(b)}|z-w| \leq \sup _{z \in \sigma(a-b)}|z|
$$


and by symmetry the proposition is proved.

The next theorem says what happens when one has a norm convergent sequence in $L(X)^{n}$. Notice that if $\sigma(a)=s p(a)$ then the conclusion would be that $f\left(a_{k}\right) \rightarrow f\left(a_{0}\right)$ in operator norm.

THeOREm 2.7. Suppose that $a_{k} \in L(X)^{n}$ are commuting tuples (not necessarily commuting with each other) for $k \geq 0$ and that $\left\|a_{k}-a_{0}\right\| \rightarrow 0$ as $k \rightarrow \infty$. If $f$ is holomorphic in a neighborhood of $\cup_{k \geq 0} \sigma\left(a_{k}\right)$, then $f\left(a_{k}\right) x \rightarrow f\left(a_{0}\right) x$ for every $x \in X$.

Proof. Consider the Banach space

$$
c(X)=\left\{\left(x_{k}\right)_{k=0}^{\infty}: \lim _{k \rightarrow \infty}\left\|x_{k}-x_{0}\right\|=0\right\}
$$

with norm $\left\|\left(x_{k}\right)_{k=0}^{\infty}\right\|_{\infty}=\sup _{k \geq 0}\left\|x_{k}\right\|$ and the tuple of $n$ operators $a^{\prime} \in$ $L(c(X))^{n}$ defined by $a^{\prime}\left(x_{k}\right)_{k=0}^{\infty}=\left(a_{k} x_{k}\right)_{k=0}^{\infty}$. Suppose that $a_{k}$ is a nonsingular tuple for every $k \geq 0$. Let $f$ be a closed $c(X)$-form, that is $\delta_{a^{\prime}} f=0$. Then $\delta_{a_{k}} f_{k}=0$ for every $k \geq 0$. Hence there is a solution $u_{0}$ of the equation $\delta_{a_{0}} u_{0}=f_{0}$ since $a_{0}$ is nonsingular. Lemma 1.3 gives a uniform constant $C$ and $v_{k}$ such that $\delta_{a_{k}} v_{k}=\delta_{a_{k}} u_{0}-f_{k}$ and

$$
\left\|v_{k}\right\| \leq C\left\|\delta_{a_{k}} u_{0}-f_{k}\right\| \leq C\left\|\delta_{a_{k}}-\delta_{a_{0}}\right\|\left\|u_{0}\right\|+C\left\|f_{0}-f_{k}\right\| .
$$

Thus $u_{k}=u_{0}-v_{k}$ solve the equations $\delta_{a_{k}} u_{k}=f_{k}$ and $u_{k} \rightarrow u_{0}$ if $k \rightarrow \infty$. Hence $u=\left(u_{k}\right)_{k=0}^{\infty}$ is a solution of $\delta_{a^{\prime}} u=f$ and the complex $K_{\bullet}\left(a^{\prime}, c(X)\right)$ is exact, and thus $a^{\prime}$ is nonsingular. That is, we have proved the inclusion

$$
\sigma\left(a^{\prime}\right) \subset \bigcup_{k \geq 0} \sigma\left(a_{k}\right)
$$

Let $u_{i}$ be smooth $c(X)$-forms defined on $C^{n} \backslash \sigma\left(a^{\prime}\right)$ by the equations

$$
\delta_{z-a^{\prime}} u_{1}=x, \quad \delta_{z-a^{\prime}} u_{i+1}=\bar{\partial} u_{i} .
$$

Thus $\left(u_{n}\right)_{k}$ represent $\omega_{z-a_{k}} x$ for all $k>0$ and $\left(u_{n}\right)_{0}=\lim _{k \rightarrow \infty}\left(u_{n}\right)_{k}$ represents $\omega_{z-a_{0}} x$. Suppose that $\phi \in C_{c}^{\infty}$ is equal to 1 in a neighborhood the union of $\sigma\left(a_{k}\right)$. Then

$$
\lim _{k \rightarrow \infty} f\left(a_{k}\right) x=-\lim _{k \rightarrow \infty} \int f \bar{\partial} \phi \wedge\left(u_{n}\right)_{k}=-\int f \bar{\partial} \phi \wedge\left(u_{n}\right)_{0}=f\left(a_{0}\right) x
$$

for all $x \in X$, and hence the theorem is proved. 


\section{Non-holomorphic functional calculus}

In this section we will extend the holomorphic functional calculus of Section 2 to functions such that $|\bar{\partial} f(z)|$ tends to zero when $z$ approaches the spectrum. If $f$ is a $C^{1}$-function with compact support, we define whenever possible

$$
f(a) x=-\int \bar{\partial} f \wedge u_{n}^{x},
$$

where $u_{n}^{x}$ is a form that represents $\omega_{z-a} x$.

Several problems occur. There is a problem with the possible dependence of the choice of representative $u_{n}^{x}$ of the class $\omega_{z-a} x$. Other problems are to investigate whether

$$
\begin{aligned}
& f(a) \in(a)^{\prime \prime}, \quad f(a) g(a)=f g(a), \\
& \sigma(f(a))=f(\sigma(a)), \quad g(f(a))=g \circ f(a)
\end{aligned}
$$

and whether $f(a)=0$ if $f=0$ on $\sigma(a)$. We will prove that $f(a) g(a)=$ $f g(a), f(a) \in(a)^{\prime \prime}$ and $\sigma(f(a))=f(\sigma(a))$ for a certain algebra $S_{a}(3.7)$ of functions. In order to do this, we will need a slightly stronger condition on $\bar{\partial} f$ than in the case $n=1$. To begin with, we will see what is needed for the muliplicative property to hold.

Suppose that $E \supset \sigma(a)$ is a compact set such that there exists a sequence $u_{i}$ on $\mathrm{C}^{n} \backslash E$ satisfying (2.4). Then we have that $u_{n}$ is operator valued and represents $\omega_{z-a}$ in $C^{n} \backslash E$. The definition of $f(a)$ in this case is

$$
f(a)=-\int \bar{\partial} f \wedge u_{n} .
$$

Define a sequence $c_{l}$ by

$$
c_{1}=0, \quad \delta_{z-a, w-a} c_{l+1}=\bar{\partial} c_{l}+u_{l}^{2}-u_{l}^{1},
$$

where $u_{l}^{1}=\pi_{1}^{*} u_{l}$ and $u_{l}^{2}=\pi_{2}^{*} u_{l}$. Thus $c_{2 n}$ represents $\omega_{z-a} \wedge \omega_{w-a}$. We now prove the multiplicative property.

Proposition 3.1. Let $u_{i}$ be a sequence defined on $C^{n} \backslash E$, where $E \supset \sigma(a)$ is a compact set, as in (2.4), and suppose that $c_{l}, n \leq l \leq 2 n$, are forms that satisfy the conditions

$$
i^{*} c_{n}=0, \quad \delta_{z-a, w-a} c_{l+1}=\bar{\partial} c_{l}+u_{l}^{2}-u_{l}^{1}, \quad c_{2 n}=u_{n}^{1} \wedge u_{n}^{2},
$$

where $i(\tau)=(\tau, \tau)$. Moreover suppose that $f, g \in C_{c}^{2}$ such that

$$
\int\left\|\bar{\partial} f \wedge u_{n}\right\|<\infty, \quad \int\left\|\bar{\partial} g \wedge u_{n}\right\|<\infty
$$


and

$$
\int_{z} \int_{w} \frac{\left\|\bar{\partial} f(z) \wedge \bar{\partial} g(w) \wedge c_{l}\right\|}{d(z, E) d(w, E)|z-w|^{2(2 n-l)-1}}<\infty,
$$

for all $l$ such that $n \leq l<2 n$. Then $f(a) g(a)=f g(a)$.

Proof. First note that

$$
f(a) g(a)=-\int_{z} \int_{w} \bar{\partial} f(z) \wedge \bar{\partial} g(w) \wedge u_{n}^{1} \wedge u_{n}^{2}
$$

and that, by the Bochner-Martinelli integral formula,

$$
\begin{aligned}
f g(a)= & -\int(g \bar{\partial} f+f \bar{\partial} g) \wedge u_{n} \\
= & \int_{z} \int_{w} \bar{\partial} f(z) \wedge \bar{\partial} g(w) \wedge m_{n} \wedge u_{n}^{1} \\
& \quad-\int_{z} \int_{w} \bar{\partial} f(z) \wedge \bar{\partial} g(w) \wedge m_{n} \wedge u_{n}^{2} .
\end{aligned}
$$

Let $\chi_{\varepsilon}$ be the convolution of the characteristic function of the set

$$
\left\{(z, w): d\left((z, w), E \times C^{n} \cup C^{n} \times E\right) \geq 2 \varepsilon\right\}
$$

and the function $\varepsilon^{-4 n} \rho(\cdot / \varepsilon)$, where $\rho$ is a non-negative smooth function with compact support in the unit ball of $C^{2 n}$ such that its integral is equal to 1 . Since

$$
\left\|\bar{\partial} f(z) \wedge \bar{\partial} g(w) \wedge\left(u_{n}^{1} \wedge u_{n}^{2}+m_{n} \wedge u_{n}^{1}-m_{n} \wedge u_{n}^{2}\right)\right\|
$$

is integrable, we must prove that

$$
\lim _{\varepsilon \rightarrow 0} \int_{z} \int_{w} \chi_{\varepsilon} \bar{\partial} f(z) \wedge \bar{\partial} g(w) \wedge\left(u_{n}^{1} \wedge u_{n}^{2}+m_{n} \wedge u_{n}^{1}-m_{n} \wedge u_{n}^{2}\right)=0 .
$$

The resolvent identity (2.10) gives that

$$
-\bar{\partial} \sum_{k+l=2 n} m_{k} \wedge c_{l}+[\Delta] \wedge c_{n}=u_{n}^{1} \wedge u_{n}^{2}+m_{n} \wedge u_{n}^{1}-m_{n} \wedge u_{n}^{2}
$$

in the sense of currents (note that the proof of this formula only made use of the forms $c_{l}$ for $l \geq n$ ). Hence, since $i^{*} c_{n}=0$, we must prove that

$$
\lim _{\varepsilon \rightarrow 0} \int_{z} \int_{w} \chi_{\varepsilon} \bar{\partial} f(z) \wedge \bar{\partial} g(w) \wedge \bar{\partial} \sum_{k+l=2 n} m_{k} \wedge c_{l}=0 .
$$


Integration by parts gives that (3.4) is equivalent to

$$
\lim _{\varepsilon \rightarrow 0} \int_{z} \int_{w} \bar{\partial} \chi_{\varepsilon} \wedge \bar{\partial} f(z) \wedge \bar{\partial} g(w) \wedge \sum_{k+l=n} m_{k} \wedge c_{l}=0 .
$$

Note that $\left|\bar{\partial} \chi_{\varepsilon}\right| \leq C \varepsilon^{-1}$ and that $\left|\bar{\partial} \chi_{\varepsilon}\right|$ has support in

$$
\varepsilon \leq d\left((z, w), E \times C^{n} \cup C^{n} \times E\right) \leq 3 \varepsilon .
$$

We also note that

$$
\begin{gathered}
d\left((z, w), E \times C^{n} \cup C^{n} \times E\right) \geq \min \{d(z, E), d(w, E)\} \\
\geq C d(z, E) d(w, E)
\end{gathered}
$$

on a bounded set, where $C>0$ is a constant (depending on the bound). Thus (3.5) follows since

$$
\int_{z} \int_{w} \frac{\left\|\bar{\partial} f(z) \wedge \bar{\partial} g(w) \wedge \sum_{k+l=2 n} m_{k} \wedge c_{l}\right\|}{d\left((z, w), E \times C^{n} \cup C^{n} \times E\right)}<\infty
$$

by (3.3). Hence the proposition is proved.

To be able to separate the condition (3.3) we will assume that $u_{i}$ commute with $a$. We can then choose the sequence $c_{i}$ in the following way.

Proposition 3.2. Suppose that $u_{i}$ is a sequence as in (2.4) and that a $u_{i}=$ $u_{i}$ a. Then

$$
c_{i}=\sum_{k+l=i} u_{k}^{1} \wedge u_{l}^{2}
$$

satisfies (3.1).

Proof. We have that $c_{1}=0$, and since $a$ and $u_{i}$ commute,

$$
\begin{aligned}
\delta c_{i+1}-\bar{\partial} c_{i}=\sum_{k+l=i+1}\left(\delta u_{k}^{1}\right. & \left.\wedge u_{l}^{2}-u_{k}^{1} \wedge \delta u_{l}^{2}\right) \\
& -\sum_{k+l=i}\left(\delta u_{k+1}^{1} \wedge u_{l}^{2}-u_{k}^{1} \wedge \delta u_{l+1}^{2}\right)=u_{i}^{2}-u_{i}^{1},
\end{aligned}
$$

where $\delta=\delta_{z-a, w-a}$. Thus $c_{i}$ satisfies (3.1).

Unfortunately, the sequence $c_{i}$ in Proposition 3.2 does not necessarily satisfy $i^{*} c_{n}=0$. However, by the proof of Theorem 2.3 we infer that $i^{*} c_{n}$ is exact. 
We have an explicit choice of sequence that satisfies (2.4). Suppose that $s$ satisfies the equalities $\delta_{z-a} s=e$ and $a s=s a$. Then

$$
\delta_{z-a} s=e, \quad \delta_{z-a}\left(s \wedge(\bar{\partial} s)^{i}\right)=(\bar{\partial} s)^{i}=\bar{\partial}\left(s \wedge(\bar{\partial} s)^{i-1}\right)
$$

and hence $u_{i}=s \wedge(\bar{\partial} s)^{i-1}$ satisfies (2.4). The sequence $c_{i}$ of Proposition 3.2 is then

$$
c_{i}=\sum_{k+l=i} s^{1} \wedge\left(\bar{\partial} s^{1}\right)^{k-1} \wedge s^{2} \wedge\left(\bar{\partial} s^{2}\right)^{l-1},
$$

where $s^{1}=\pi_{1}^{*} s$ and $s^{2}=\pi_{2}^{*} s$. Note that if $s \wedge s=0$ then $s \wedge(\bar{\partial} s)=(\bar{\partial} s) \wedge s$ and hence $i^{*} c_{n}=0$.

Let $E \supset \sigma(a)$ be a compact set and let $s$ be a given form such that $s$ is defined on $C^{n} \backslash E, \delta_{z-a} s=e$ and $a s=s a$. Define the class $S_{a}$ by

$$
S_{a}=\left\{f \in C_{c}^{2}\left(C^{n}\right):\|f\|_{a}<\infty\right\},
$$

where

$$
\begin{aligned}
&\|f\|_{a}=\sum_{i=1}^{n}\left\|\frac{\bar{\partial} f \wedge s \wedge(\bar{\partial} s)^{i-1}}{d(z, E)}\right\|_{\infty} \\
& \quad+\sum_{k+l=n}\left\|\frac{\bar{\partial} f \wedge s \wedge(\bar{\partial} s)^{k-1} \wedge s \wedge(\bar{\partial} s)^{l-1}}{d(z, E)}\right\|_{\infty} .
\end{aligned}
$$

Note that the second sum vanishes if $s \wedge s=0$. This is always the case if $n=2$ since then $\delta_{z-a}(s \wedge s)=s-s=0$ and $\delta_{z-a}$ injective. If $n=1$ then $S_{a}$ defined by (3.7) is a slightly smaller class than $S_{a}$ defined in the introduction. This is because the left hand side in the resolvent identity (2.10) is 0 if $n=1$. If $f \in S_{a}$ then $f(a)$ is defined by

$$
f(a)=-\int \bar{\partial} f \wedge s \wedge(\bar{\partial} s)^{n-1} .
$$

Of course we have that $f(a) \in L(X)$ if $f \in S_{a}$. Note that $S_{a}$ is an algebra. In the next lemma we will use Proposition 3.1 to prove that $f(a) g(a)=f g(a)$ if $f, g \in S_{a}$.

Lemma 3.3. If $f, g \in S_{a}$ then $f(a) g(a)=f g(a)$.

Proof. Let $c_{i}$ be the sequence defined by (3.6) and let

$$
d_{i}=\sum_{k+l=i} s^{2} \wedge\left(\bar{\partial} s^{2}\right)^{k-1} \wedge s^{2} \wedge\left(\bar{\partial} s^{2}\right)^{l-1} .
$$


By a computation similar to the proof of Proposition 3.2, we see that the sequence $d_{i}$ satisfies the relation

$$
\delta_{z-a, w-a} d_{i+1}=\bar{\partial} d_{i},
$$

and hence that $\bar{\partial} d_{n}=0$. For every $l>n$ define $c_{l}^{\prime}$ by $c_{l}^{\prime}=c_{l}$ and define $c_{n}^{\prime}$ by $c_{n}^{\prime}=c_{n}-d_{n}$. Then $c_{l}^{\prime}$ satisfies the condition (3.2) since $\bar{\partial} d_{n}=0$ and $i^{*} c_{n}=i^{*} d_{n}$. We have that $|z-w|^{-2 n+1}$ is a locally integrable function on $\mathrm{C}^{2 n}$ and hence

$$
\begin{aligned}
& \int_{z} \int_{w} \frac{\left\|\bar{\partial} f(z) \wedge \bar{\partial} g(w) \wedge c_{i}\right\|}{d(z, E) d(w, E)|z-w|^{2 n-1}} \\
& \quad \leq \sum_{k+l=i} \int_{z} \int_{w} \frac{\left\|\bar{\partial} f(z) \wedge s^{1} \wedge\left(\bar{\partial} s^{1}\right)^{k-1}\right\|\left\|\bar{\partial} g(w) \wedge s^{2} \wedge\left(\bar{\partial} s^{2}\right)^{l-1}\right\|}{d(z, E) d(w, E)|z-w|^{2 n-1}}<\infty .
\end{aligned}
$$

Similarly, we remark that

$$
\int_{z} \int_{w} \frac{\left\|\bar{\partial} f(z) \wedge \bar{\partial} g(w) \wedge d_{n}\right\|}{d(z, E) d(w, E)|z-w|^{2 n-1}}<\infty,
$$

since $\|g\|_{a}<\infty$. Thus the statement follows from Proposition 3.1.

In order to prove that $f(a) \in(a)^{\prime \prime}$ we construct the resolvent $\omega_{z-a, w-b}$ and use the multiplicative property of the functional calculus of the tuple $(a, b)$, where $b \in L(X)$ commutes with $a$.

Lemma 3.4. If $f \in S_{a}$, then $f(a) \in(a)^{\prime \prime}$.

Proof. Suppose that $b \in L(X)$ is an operator such that $a b=b a$. Define the form

$$
v(w)=\frac{1}{2 \pi i}(w-b)^{-1} d w
$$

Define the sequence $c_{k}$ by

$$
c_{1}=0, \quad c_{k}=v \wedge s \wedge(\bar{\partial} s)^{k-2} .
$$

Then we have the equations

$$
c_{1}=0, \quad \delta_{z-a, w-b} c_{2}=s-v
$$

and

$$
\delta_{z-a, w-b} c_{k+1}=s \wedge(\bar{\partial} s)^{k-1}-v \wedge(\bar{\partial} s)^{k-1}=\bar{\partial} c_{k}+s \wedge(\bar{\partial} s)^{k-1} .
$$


Let $\chi$ be a smooth cutoff function such that $\{\chi, 1-\chi\}$ is a partition of unity subordinate the cover

$$
\{\{(z, w): z \notin E,|w|<3\|b\|\},\{(z, w):|w|>2\|b\|\}\}
$$

of $C^{n} \times C \backslash E \times\{w:|w| \leq 2\|b\|\}$. This is a special choice of function $\chi$ used in Lemma 3.2 of [1] which enables us to avoid an integration by parts. Define the sequence $a_{k}$ outside $E \times\{w:|w| \leq 2\|b\|\}$ by

$$
a_{1}=\chi s+(1-\chi) v, \quad a_{k}=\chi s \wedge(\bar{\partial} s)^{k-1}-\bar{\partial} \chi \wedge c_{k} .
$$

We then have that

$$
\delta_{z-a, w-b} a_{1}=e, \quad \delta_{z-a, w-b} a_{2}=\chi \bar{\partial} s+\bar{\partial} \chi \wedge(s-v)=\bar{\partial} a_{1}
$$

and that

$$
\delta_{z-a, w-b} a_{k+1}=\chi(\bar{\partial} s)^{k}+\bar{\partial} \chi \wedge\left(\bar{\partial} c_{k}+s \wedge(\bar{\partial} s)^{k-1}\right)=\bar{\partial} a_{k},
$$

and thus

$$
a_{n+1}=-\bar{\partial} \chi \wedge v \wedge s \wedge(\bar{\partial} s)^{n-1}
$$

represents $\omega_{z-a, w-b}$. Choose $\phi \in C_{c}^{\infty}(\mathbf{C})$ which is 1 in a neighborhood of $\{w \in \mathrm{C}:|w|<3\|b\|\}$. Then we have that

$$
\begin{aligned}
(\phi f)(a, b)= & -\int_{w} \int_{z} \bar{\partial}(\phi(w) f(z)) \wedge a_{n+1}(z, w) \\
= & \iint f \bar{\partial}_{w} \phi \wedge \bar{\partial}_{z} \chi \wedge v \wedge s \wedge(\bar{\partial} s)^{n-1} \\
& \quad+\iint \phi \bar{\partial}_{z} f \wedge \bar{\partial}_{w} \chi \wedge v \wedge s \wedge(\bar{\partial} s)^{n-1} \\
= & -\int \bar{\partial} f \wedge s \wedge(\bar{\partial} s)^{n-1}=f(a) .
\end{aligned}
$$

Let $a_{k}^{1}=\pi_{1}^{*} a_{k}$ and $a_{k}^{2}=\pi_{2}^{*} a_{k}$, where

$$
\pi_{1}\left(z_{1}, w_{1}, z_{2}, w_{2}\right)=\left(z_{1}, w_{1}\right) \quad \text { and } \quad \pi_{2}\left(z_{1}, w_{1}, z_{2}, w_{2}\right)=\left(z_{2}, w_{2}\right) .
$$

Define the sequence $c_{i}^{\prime}$ by

$$
c_{1}^{\prime}=0, \quad c_{i}^{\prime}=\sum_{k+l=i} a_{k}^{1} \wedge a_{l}^{2}
$$


so that by Proposition 3.2,

$$
c_{1}=0, \quad \delta_{z_{1}-a, w_{1}-b, z_{2}-a, w_{2}-b} c_{i+1}^{\prime}=\bar{\partial} c_{i}^{\prime}+a_{i}^{2}-a_{i}^{1} .
$$

Let $F=E \times\{w:|w| \leq 2\|b\|\}$. Define the function $g$ by $g(z, w)=w \psi(z, w)$ where $\psi \in C_{c}^{\infty}$ is equal to 1 in a neighborhood of $F$. We have that

$$
\begin{aligned}
\left\|\frac{\bar{\partial}(\phi f) \wedge a_{k}}{d((z, w), F)}\right\|_{\infty} \leq\left\|\frac{\chi \bar{\partial}(\phi f) \wedge s \wedge(\bar{\partial} s)^{k-1}}{d(z, E)}\right\|_{\infty} \\
+\left\|\frac{\bar{\partial}(\phi f) \wedge \bar{\partial} \chi \wedge v \wedge s \wedge(\bar{\partial} s)^{k-2}}{d(z, E)}\right\|_{\infty}<\infty
\end{aligned}
$$

since $f \in S_{a}$. Hence we have that

$$
\iint \frac{\left\|\bar{\partial}\left(\phi\left(w_{1}\right) f\left(z_{1}\right)\right) \wedge \bar{\partial} g\left(z_{2}, w_{2}\right) \wedge c_{l}^{\prime}\right\|}{d\left(\left(z_{1}, w_{1}\right), F\right) d\left(\left(z_{2}, w_{2}\right), F\right)\left|\left(z_{1}, w_{1}\right)-\left(z_{2}, w_{2}\right)\right|^{2 n+1}}<\infty
$$

for all $l$. Define the forms $c_{l}^{\prime \prime}$ by the equations $c_{l}^{\prime \prime}=c_{l}^{\prime}$ if $l>n+1$ and

$$
c_{n+1}^{\prime \prime}=c_{n+1}^{\prime}-\sum_{k+l=n+1} a_{k}^{2} \wedge a_{l}^{2} .
$$

Then we have that $c_{n+1}^{\prime \prime}$ satisfies $i^{*} c_{n+1}^{\prime \prime}=0$ and hence by Proposition 3.1 we have that $(\phi f)(a, b) g(a, b)=g(a, b)(\phi f)(a, b)$ since

$$
\iint \frac{\left\|\bar{\partial}\left(\phi\left(w_{1}\right) f\left(z_{1}\right)\right) \wedge \bar{\partial} g\left(z_{2}, w_{2}\right) \wedge \sum_{k+l=n+1} a_{k}^{2} \wedge a_{l}^{2}\right\|}{d\left(\left(z_{1}, w_{1}\right), F\right) d\left(\left(z_{2}, w_{2}\right), F\right)\left|\left(z_{1}, w_{1}\right)-\left(z_{2}, w_{2}\right)\right|^{2 n+1}}<\infty .
$$

Thus $f(a) b=b f(a)$ since $g(a, b)=b$ by the holomorphic functional calculus.

We can now prove a generalization of the holomorphic functional calculus.

THEOREM 3.5 (Non-holomorphic functional calculus). Suppose that a is an n-tuple of commuting operators and that $E \supset \sigma(a)$ is compact such that it exists a smooth form s defined on $\mathrm{C}^{n} \backslash E$ with $\delta_{z-a} s=e$ and as $=$ sa. Let $S_{a}$ be the class defined by (3.7) and let $f(a), f \in S_{a}$, be the operator defined by

$$
f(a)=-\int \bar{\partial} f \wedge s \wedge(\bar{\partial} s)^{n-1} .
$$

Then the map $f \mapsto f(a): S_{a} \rightarrow(a)^{\prime \prime}$ is a continuous algebra homomorphism that continuously extends the map $f \mapsto f(a): \mathscr{O}(E) \rightarrow(a)^{\prime \prime}$. 
Proof. By Lemma 3.4 the map $f \mapsto f(a): S_{a} \rightarrow(a)^{\prime \prime}$ is well defined. The map is continuous and linear. Lemma 3.3 shows that the map is multiplicative, and thus the map is an algebra homomorphism. To see that it continuously extends the map $f \mapsto f(a): \mathscr{O}(E) \rightarrow(a)^{\prime \prime}$, suppose that we have a sequence $f_{n} \in \mathscr{O}(U)$, where $U$ is an open neighborhood of $E$, and that $f_{n} \rightarrow 0$ uniformly on compacts. Then

$$
\left\|f_{n} \phi\right\|_{a} \rightarrow 0
$$

where $\phi \in C_{c}^{\infty}(U)$ is a function equal to 1 in a neighborhood of $E$.

We now go on and prove the spectral mapping theorem for this functional calculus. To do this, we need the following lemma which shows that $f(w)$ acts as $f(a)$ on $H_{p}(w-a, c, X)$.

Lemma 3.6. Suppose that there is an operator valued form s outside $E$ such that $\delta_{z-a} s=e$ and $s a=$ as. Furthermore, suppose that $c \in\left((a)^{\prime \prime}\right)^{m}, w \in \sigma(a)$ and $k \in K_{p}(w-a, c, X)$ (with respect to a basis $d w_{1}, \ldots, d w_{n}, e_{n+1}, \ldots, e_{n+m}$ of $\left.\mathrm{C}^{n+m}\right)$ such that $\delta_{w-a, c} k=0$. If $f \in S_{a}$, then

$$
(f(a)-f(w)) k=\delta_{w-a, c} \int_{z} \bar{\partial} f(z) \wedge \sum_{l=1}^{n} m_{n+1-l}^{\prime \prime} \wedge s \wedge(\bar{\partial} s)^{l-1} \wedge k,
$$

where $m_{i}^{\prime \prime}$ is defined in the proof.

Proof. We have that

$$
\delta_{z-a, w-a} m_{1}=e, \quad \delta_{z-a, w-a} m_{i+1}=\bar{\partial} m_{i},
$$

by (2.7) and (2.8), where $m_{i}$ is defined by (2.6). We also have that

$$
\delta_{z-a, w-a} s=e, \quad \delta_{z-a, w-a}\left(s \wedge(\bar{\partial} s)^{i}\right)=\bar{\partial}_{z}\left(s \wedge(\bar{\partial} s)^{i-1}\right),
$$

where $s$ only depends on $z$. Therefore the same calculation as in the proof of Proposition 3.2 shows that

$$
\delta_{z-a, w-a} \sum_{k+l=i+1} m_{k} \wedge s \wedge(\bar{\partial} s)^{l-1}-\bar{\partial} \sum_{k+l=i} m_{k} \wedge s \wedge(\bar{\partial} s)^{l-1}=s \wedge(\bar{\partial} s)^{i-1}-m_{i} .
$$

Let $i=n$ and identify the component without any $d w$ and $d \bar{w}$ in this expression to get,

$$
\delta_{w-a} \sum_{k+l=n+1} m_{k}^{\prime \prime} \wedge s \wedge(\bar{\partial} s)^{l-1}=s \wedge(\bar{\partial} s)^{n-1}-m_{n}^{\prime}+\bar{\partial}_{z} \sum_{k+l=n} m_{k}^{\prime} \wedge s \wedge(\bar{\partial} s)^{l-1},
$$


where

$$
m_{k}^{\prime}=\frac{1}{(2 \pi i)^{k}} \frac{\partial_{z}|z-w|^{2}}{|z-w|^{2}} \wedge\left(\bar{\partial}_{z} \frac{\partial_{z}|z-w|^{2}}{|z-w|^{2}}\right)^{k-1}
$$

and $m_{k}^{\prime \prime}$ is the component of $m_{k}$ with one $d w$ and no $d \bar{w}$. Let $\chi_{\varepsilon}$ be the convolution of the characteristic function of the set

$$
\{z: d(z, E) \geq 2 \varepsilon\}
$$

and the function $\varepsilon^{-2 n} \rho(\cdot / \varepsilon)$, where $\rho$ is a non-negative smooth function with compact support in the unit ball of $\mathrm{C}^{n}$ such that its integral is equal to 1 . We have that

$$
\begin{aligned}
& \int_{z} \bar{\partial}_{z} f(z) \wedge \bar{\partial}_{z} \sum_{k+l=n} m_{k}^{\prime} \wedge s \wedge(\bar{\partial} s)^{l-1} \\
&=\lim _{\varepsilon \rightarrow 0} \int_{z} \chi_{\varepsilon} \bar{\partial}_{z} f(z) \wedge \bar{\partial}_{z} \sum_{k+l=n} m_{k}^{\prime} \wedge s \wedge(\bar{\partial} s)^{l-1} \\
& \quad=\lim _{\varepsilon \rightarrow 0} \int_{z} \bar{\partial}_{z} \chi_{\varepsilon} \wedge \bar{\partial}_{z} f(z) \wedge \sum_{k+l=n} m_{k}^{\prime} \wedge s \wedge(\bar{\partial} s)^{l-1}=0
\end{aligned}
$$

since $\left|\bar{\partial} \chi_{\varepsilon}\right| \leq C \varepsilon^{-1}$ and $\left|\bar{\partial} \chi_{\varepsilon}\right|$ has support in $\varepsilon \leq d(z, E) \leq 3 \varepsilon$. Hence we have that

$$
\begin{aligned}
f(a)-f(w) & =\int_{z} \bar{\partial} f(z) \wedge\left(s \wedge(\bar{\partial} s)^{n-1}-m_{n}^{\prime}\right) \\
& =\delta_{w-a} \int_{z} \bar{\partial} f(z) \wedge \sum_{k+l=n+1} m_{k}^{\prime \prime} \wedge s \wedge(\bar{\partial} s)^{l-1} .
\end{aligned}
$$

Therefore,

$$
(f(a)-f(w)) k=\delta_{w-a, c} \int_{z} \bar{\partial} f(z) \wedge \sum_{l=1}^{n} m_{n+1-l}^{\prime \prime} \wedge s \wedge(\bar{\partial} s)^{l-1} \wedge k,
$$

since $(w-a, c)$ and $s$ commute.

We can now prove the spectral mapping theorem.

THEOREM 3.7 (Spectral mapping theorem). If $f$ is tuple of functions in $S_{a}$, where $S_{a}$ is defined by (3.7), then $\sigma(f(a))=f(\sigma(a))$.

Proof. Suppose that we can prove the statement; if $z \in \sigma(a)$ then $(z-$ $a, f(a))$ is nonsingular if and only if $f(z) \neq 0$. In that case $(z-a, w-f(a))$ is nonsingular if and only if $w-f(z) \neq 0$ and hence $\sigma(f(a))=\pi_{2} \sigma(a, f(a))=\pi_{2}\{(z, w): w=f(z), z \in \sigma(a)\}=f(\sigma(a))$ 
by Theorem 1.4.

Suppose that $z \in \sigma(a)$. We have the induction hypothesis that if $m$ is a natural number then the tuple $(z-a, f(a))$ is nonsingular if and only if $f(z) \neq 0$ for all $m$-tuples $f$ of functions in $S_{a}$. The case $m=0$ follows from Lemma 3.6. Assume that the hypothesis has been proved for $m$. Given $f^{\prime}=\left(f_{1}, \ldots, f_{m+1}\right)$ let $f=\left(f_{1}, \ldots, f_{m}\right)$. Then there is a long exact sequence

$\ldots \rightarrow H_{p}(z-a, f(a), X) \rightarrow H_{p}\left(z-a, f^{\prime}(a), X\right)$

$$
\rightarrow H_{p-1}(z-a, f(a), X) \stackrel{f_{m+1}(a)}{\longrightarrow} H_{p-1}(z-a, f(a), X) \rightarrow \ldots,
$$

for this see Taylor [15], Lemma 1.3. Lemma 3.6 gives that the last homomorphism is equal to $f_{m+1}(z)$. Hence

$$
H_{p}\left(z-a, f^{\prime}(a), X\right)=0
$$

if $f_{m+1}(z) \neq 0$ and

$$
\Im H_{p}\left(z-a, f^{\prime}(a), X\right)=H_{p-1}(z-a, f(a), X)
$$

if $f_{m+1}(z)=0$. Therefore the induction hypothesis hold for $m+1$ and hence the theorem follows.

We now consider a case where we can answer all the question we set up in the beginning of this section. Denote by $\operatorname{co}(E)$ the convex hull of the set $E$.

THEOREM 3.8. Let $h$ be a positive decreasing function on $[0, \infty)$. If there is a differential form $u^{x}$ on $\mathrm{C}^{n} \backslash \operatorname{co}(\sigma(a))$ such that $\left\|u^{x}(z)\right\| \leq\|x\| h(d(z, E))$ then we define the class $S_{h}(a)$ by

$$
S_{h}(a)=\left\{f \in C_{c}^{1}\left(C^{n}\right):\||\bar{\partial} f(z)| h(d(z, c o(\sigma(a))))\|_{L^{\infty}}<\infty\right\} .
$$

Let the norm of functions in $S_{h}(a)$ be given by

$$
\|f\|_{S_{h}(a)}=\||\bar{\partial} f(z)| h(d(z, \operatorname{co}(\sigma(a))))\|_{L^{1}} .
$$

Then the map

$$
f \mapsto f(a): S_{h}(a) \rightarrow(a), \quad \text { where } \quad f(a) x=-\int \bar{\partial} f \wedge u^{x},
$$

is a continuous algebra homomorphism. If $f \in S_{h}(a)$ then $\sigma(f(a))=f(\sigma(a))$ and $f(a)=0$ if $f=0$ on $\operatorname{co}(\sigma(a))$. Furthermore, if $f \in S_{h}(a), g \in$ $S_{h_{1}}(f(a))$ (or $\left.g \in \mathscr{O}(\sigma(f(a)))\right)$, where $h_{1}$ is a decreasing function such that $h(y / \sup |d f|) \leq C h_{1}(y), y \in[0, \infty)$, and $g(0)=0$ then $g(f(a))=g \circ f(a)$. 
Proof. Suppose that $0 \in \operatorname{co}(\sigma(a))$ and let $f_{t}(z)=f(t z)$ for $t<1$. Since $h\left(d(z, \operatorname{co}(\sigma(a))) \leq h\left(d(t z, \operatorname{co}(\sigma(a)))\right.\right.$ we see that $f_{t} \rightarrow f$ in $S_{h}(a)$ by dominated convergence. We know that all the conclusions in the theorem holds for functions that are holomorphic in a neighborhood of the spectrum. Since $\|f(a)\|_{L(X)} \leq\|f\|_{S_{h}(a)}$ we will be able to prove the theorem using the approximation $f_{t}$. Consider especially the spectral mapping property. By Proposition $2.6 \sigma\left(f_{t}(a)\right)$ deforms continuously to $\sigma(f(a))$. Since $f_{t}(\sigma(a))$ also deforms continuously to $f(\sigma(a))$, we get $\sigma(f(a))=f(\sigma(a))$. For the composition rule we have

$$
\begin{aligned}
\|g(f(a)) x-g \circ f(a) x\| \leq \| & (f(a)) x-g_{s}(f(a)) x \| \\
+ & \left\|g_{s}(f(a)) x-g_{s}\left(f_{t}(a)\right) x\right\| \\
& +\left\|g_{s} \circ f_{t}(a) x-g_{s} \circ f(a) x\right\| \\
& +\left\|g_{s} \circ f(a) x-g \circ f(a) x\right\| \\
\leq \| & -g_{s}\left\|_{S_{h_{1}}(f(a))}\right\| x \| \\
& +\left\|g_{s}(f(a)) x-g_{s}\left(f_{t}(a)\right) x\right\| \\
& +\left\|g_{s} \circ f_{t}-g_{s} \circ f\right\|_{S_{h}(a)}\|x\| \\
& +\left\|g_{s} \circ f-g \circ f\right\|_{S_{h}(a)}\|x\| \rightarrow 0,
\end{aligned}
$$

by Theorem 2.7 and

$$
\begin{aligned}
&\left|\bar{\partial}\left(g_{s} \circ f-g \circ f\right)\right| h(d(z, \operatorname{co}(\sigma(a)))) \\
& \leq\left|\partial\left(g_{s}-g\right)\right||\bar{\partial} f| h(d(z, \operatorname{co}(\sigma(a)))) \\
& \quad+\left|\bar{\partial}\left(g_{s}-g\right)\right||\partial f| h_{1}(d(f(z), \operatorname{co}(f(\sigma(a))))) .
\end{aligned}
$$

\section{REFERENCES}

1. Andersson, M., Taylor's functional calculus for commuting operators with Cauchy-FantappieLeray formulas, Internat. Math. Res. Notices 6 (1997), 247-258.

2. Andersson, M., Correction to "Taylor's functional calculus for commuting operators with Cauchy-Fantappie-Leray formulas", Internat. Math. Res. Notices 2 (1998), 123-124.

3. Andersson, M., and Berndtsson, B., Non-holomorphic functional calculus for commuting operators on a Banach space with real spectrum, In preparation.

4. Andersson, M., and Sandberg, S., A constructive proof of the composition rule for Taylors's functional calculus, Studia Math. 142 (2000), 65-69.

5. Droste, B., Extension of analytic functional calculus mappings and duality by $\bar{\partial}$-closed forms with growth, Math. Ann. 261 (1982), 185-200.

6. Dynkin, E. M., An operator calculus based on the Cauchy-Green formula (Russian), Zap. Nauchn. Sem. Leningrad. Otdel. Mat. Inst. Steklov 30 (1972), 33-39. 
7. Eschmeier, J., and Putinar, M., Spectral decompositions and analytic sheaves, London Math. Soc. Monographs (N.S) 10 (1996).

8. Harte, R., Invertibility and singularity for bounded linear operators, Monographs Textbooks Pure Appl. Math. 109 (1988).

9. Kisil, V. V., and Ramirez de Arellano, E., The Riesz-Clifford functional calculus for noncommuting operators and quantum field theory, Math. Methods Appl. Sci. 19 (1996), no. 8, 593-605.

10. Krantz, S., Function theory of several complex variables, Pure and applied mathematics, John Wiley \& Sons, Inc., New York (1982).

11. Müller, V., The splitting spectrum differs from the Taylor spectrum, Studia Math. 123 (1997), no. 3, 291-294.

12. Newburgh, J. D., The variation of spectra, Duke Math. J. 18 (1951), 165-176.

13. Nguyen, T. H., Calcul fonctionnel dpéndant de la croissance des coefficients spectraux, Ann. Inst. Fourier 27 (1977), no. 4, 169-199.

14. Range, R. M., Holomorphic functions and integral representations in several complex variables, Graduate Texts in Math. 108 (1986).

15. Taylor, J. L., A joint spectrum for several commuting operators, J. Funct. Anal. 6 (1970), $172-191$.

16. Taylor, J. L., The analytic-functional calculus for several commuting operators, Acta Math. 125 (1970), 1-38.

17. Taylor, J. L., Homology and cohomology for topological algebras, Adv. Math. 9 (1972), $147-182$.

18. Taylor, J. L., A general framework for a multi-operator functional calculus, Adv. Math. 9 (1972), 184-252.

DEPARTMENT OF MATHEMATICS

CHALMERS UNIVERSITY OF TECHNOLOGY AND THE UNIVERSITY OF GÖTEBORG

SE-412 96 GÖTEBORG

SWEDEN

E-mail: sebsand@math.chalmers.se 